\title{
Performance tuning of gas sensors based on epitaxial graphene on silicon carbide
}

Marius Rodner, Jasna Bahonjic, Marcus Mathisen, Rickard Gunnarsson, Sebastian Ekeroth, Ulf Helmersson, Ivan Gueorguiev Ivanov, Rositsa Yakimova and Jens Eriksson

The self-archived postprint version of this journal article is available at Linköping University Institutional Repository (DiVA):

http://urn.kb.se/resolve?urn=urn:nbn:se:liu:diva-149676

N.B.: When citing this work, cite the original publication.

Rodner, M., Bahonjic, J., Mathisen, M., Gunnarsson, R., Ekeroth, S., Helmersson, U., Ivanov, I. G., Yakimova, R., Eriksson, J., (2018), Performance tuning of gas sensors based on epitaxial graphene on silicon carbide, Materials \& design, 153, 153-158. https://doi.org/10.1016/j.matdes.2018.04.087

Original publication available at:

https://doi.org/10.1016/j.matdes.2018.04.087

Copyright: Elsevier

http://www.elsevier.com/

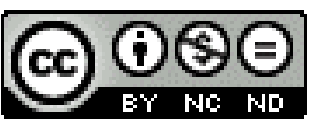




\title{
Performance tuning of gas sensors based on epitaxial graphene on silicon carbide
}

\author{
Marius Rodner ${ }^{1}$, Jasna Bahonjic ${ }^{1}$, Marcus Mathisen ${ }^{1}$, Rickard Gunnarsson ${ }^{2}$, Sebastian Ekeroth ${ }^{2}$, Ulf \\ Helmersson ${ }^{2}$, Ivan G. Ivanov ${ }^{3}$, Rositsa Yakimova ${ }^{3}$, Jens Eriksson ${ }^{1}$ \\ ${ }^{1}$ Applied Sensor Science Unit, IFM, Linköping University, Sweden \\ ${ }^{2}$ Plasma \& Coatings Physics Division, IFM, Linköping University, Sweden \\ ${ }^{3}$ Semiconductor Materials Division, IFM, Linköping University, Sweden \\ Corresponding author: Marius Rodner, marius.rodner@liu.se, +46 (0) 700896785
}

\begin{abstract}
In this study, we investigated means of performance enhancement in sensors based on epitaxial graphene on silicon carbide ( $\mathrm{SiC}$ ). Epitaxially grown graphene on SiC substrates were successfully decorated with metal oxide nanoparticles such as $\mathrm{TiO}_{2}$ and $\mathrm{Fe}_{3} \mathrm{O}_{4}$ using hollow cathode pulsed plasma sputtering. Atomic Force Microscopy and Raman data verified that no damage was added to the graphene surface. It could be shown that it was easily possible to detect benzene, which is one of the most dangerous volatile organic compounds, with the $\mathrm{Fe}_{3} \mathrm{O}_{4}$ decorated graphene sensor down to an ultra-low concentration of $5 \mathrm{ppb}$ with a signal to noise ratio of $35 \mathrm{~dB}$. Moreover, upon illumination with a UV light LED ( $265 \mathrm{~nm}$ ) of the $\mathrm{TiO}_{2}$ decorated graphene sensor, the sensitivity towards a change of oxygen could be enhanced such that a clear sensor response could be seen which is a significant improvement over dark conditions, where almost no response occurred. As the last enhancement, the time derivative sensor signal was introduced for the sensor data evaluation, testing the response towards a change of oxygen. This sensor signal evaluation approach can be used to decrease the response time of the sensor by at least one order of magnitude.
\end{abstract}

\section{Keywords}

Epitaxial graphene; metal oxide nanoparticles; gas sensor; UV light; derivative sensor signal; benzene

\section{Highlights}

Hollow cathode pulsed plasma sputtering enables deposition of $\mathrm{TiO}_{2}$ and $\mathrm{Fe}_{3} \mathrm{O}_{4}$ nanoparticles on epitaxial graphene without structural damage.

Nanoparticle decoration of epitaxial graphene yields detection of $5 \mathrm{ppb}$ benzene with a signal to noise ratio of $35 \mathrm{~dB}$.

Integration of UV LED into the sensor package expands the dynamic range for oxygen detection.

Use of time derivative signal allows reduction of response time by more than an order of magnitude. 


\section{Introduction}

Nowadays, people spend more than $85 \%$ of their time indoors, which leads to prolonged exposures towards indoor air which might be controlled towards temperature, humidity, and the ratio of oxygen and carbon dioxide, but not towards many toxic gases. Volatile organic compounds (VOCs), defined by the World Health Organization as all organic compounds having their boiling points between $50-100^{\circ} \mathrm{C}$ and $240-260^{\circ} \mathrm{C}$, are in the top 5 of global air pollutants [1]. Although some of them are hazardous to humans even at very low concentrations, they are widely used in industry and everyday products such as solvents. For example, the safety level for formaldehyde is $81 \mathrm{ppb}$, for naphthalene $1.9 \mathrm{ppb}$ and no exposure at all for benzene [2]. Moreover, indoor air pollution is in the top 5 of environmental risks to human health with 4.3 million deaths/year including 570000 children under the age of 5. This number is estimated to rise up to 6 million deaths/year in 2050 [3]. There is clearly a demand for gas sensors where ultra-high sensitivity is necessary. Until now, there are not sensors available for all the relevant gases although the marked is estimated to be $\$ 1.4$ billion by 2021 [4].

Since the isolation and first characterization of graphene in 2004, it has become the best investigated two-dimensional (2D) material with many outstanding properties like perfect thermal conductivity, ballistic electron transport at room temperature, low mass and an ultimate surface to volume ratio [5]. It can therefore be of use in very different fields of applications, one of which is as very sensitive chemical gas sensors [6]. Due to the low density of states near the Dirac point, a small change in the number of charge carriers results in a large change in the electronic state which then can easily be measured as a change in resistance of the sensor surface [7].

Epitaxial growth of graphene on silicon carbide (SiC) [8] is not the most used but a highly suitable production process when it comes to electrical devices [9]. Si atoms sublimate in argon ambient at $2000{ }^{\circ} \mathrm{C}$ and the carbon atoms self-assemble with the bulk SiC to form a monolayer of graphene with a carbon buffer layer that is formed in between graphene and the SiC interface and is still partially covalently bonded to the $\mathrm{Si}$ atoms which makes it insulating. High temperature growth leads to high quality where the thickness can be controlled by the temperature, achieving monolayer graphene with a thickness uniformity of about $98 \%$ ( $2 \%$ bilayer). Since it is grown on a semiconducting or semi insulating substrate, there is no graphene transfer needed after the growth and it can be used directly to fabricate electronic devices without short circuit effects.

Besides the sensitivity of a gas sensor, also the selectivity is of importance. Since graphene is quite sensitive to a large number of gases, there is a lack of selectivity that has to be overcome. Enhancing the sensing properties of gas sensors can be done with several different approaches. It was shown that metal- and metal oxide nanoparticles can be used effectively to enhance the selectivity of graphene, and also to generate sensitivity towards gases to which as-grown graphene does not respond [10, 11]. The addition of nanoparticles onto graphene results in specific sensor responses to different gases. The most common materials used are $\mathrm{SnO}_{2}[12,13], \mathrm{ZnO}[14,15]$ and $\mathrm{TiO}_{2}[11,16]$. Hollow cathode sputtering where the nanoparticles are grown in the gas phase is one of the most suitable techniques for scalable deposition of nanoparticles in a very ordered way on top of a surface without introducing much damage to it [17]. Another approach towards selectivity tuning is to use UV light exposure during the measurement to decrease time constants and also increase sensitivity towards certain gases by tuning the energy level [18]. In a last step, smart data processing can be used to further increase selectivity and decrease time constants if applied correctly [19]. In the following, we will introduce all of these techniques and show that they can be used to enhance the performance of graphene gas sensors. 


\section{Methods}

\subsection{Graphene growth}

As a sensor basis, epitaxial graphene with a monolayer ratio of about $98 \%$ (2 \% bilayer), grown on SiC was used. Epitaxial graphene (EG) was prepared by sublimation of $\mathrm{SiC}$ and subsequent graphene formation on semi-insulating, Si-terminated, $4 \mathrm{H}-\mathrm{SiC}(0001)$ on-axis substrates at $2000{ }^{\circ} \mathrm{C}$ in argon and at a pressure of 1 bar [20].

\subsection{Nanoparticle deposition}

$\mathrm{TiO}_{2}$ and $\mathrm{Fe}_{3} \mathrm{O}_{4}$ nanoparticles were deposited on top of the graphene layer using a hollow cathode pulsed plasma sputtering technique [21]. The nanoparticles nucleate and grow in the plasma ejected from the hollow cathode. The substrates were placed outside the dense plasma region, preventing energetic species from reaching them. The discharge parameters for the $\mathrm{Fe}_{3} \mathrm{O}_{4}$ particles were: pulse frequency $1200 \mathrm{~Hz}$, pulse width $80 \mu \mathrm{s}$ and an average power of $90 \mathrm{~W}$. A substrate bias was used to attract the particles to the substrate surface. For the $\mathrm{Fe}_{3} \mathrm{O}_{4}$ particles this bias was $+3 \mathrm{~V}$ and the substrates were exposed to nanoparticle deposition for 15 seconds. For the $\mathrm{TiO}_{2}$ nanoparticles the deposition time was 30 seconds; other process parameters are given in reference [17].

\subsection{Characterization techniques}

Before and after the deposition of nanoparticles, a series of characterization measurements were conducted to see if any damage of the graphene surface occurred during the deposition. Atomic Force Microscopy (AFM) (Quadrexed Dimension 3100 with a Nanoscope IVa controller) with a Si tip (PPPNCHR-50 from Nanosensors) and a tip radius of curvature $<7 \mathrm{~nm}$ was used in tapping mode to obtain topography images of the sensing layers. A micro Raman setup was used to perform Raman spectroscopy and reflectance measurements. A CCD camera coupled to a monochromator (HR460) was used along with a 100X magnification objective which were exited at $532 \mathrm{~nm}$ using a solid-state laser with a power of $17 \mathrm{~mW}$ focused to a spot with diameter $\sim 0.9$ um on the sample, and a system spectral resolution of approximately $5 \mathrm{~cm}^{-1}$. The Raman spectra of Si-face graphene were obtained by subtracting a reference Raman spectrum of $4 \mathrm{H}-\mathrm{SiC}$ (0001).

\subsection{Sensor device fabrication}

Sensor devices have been fabricated on SiC substrates by depositing electrodes on top of the graphene surface through thermal evaporation of titanium $(2 \mathrm{~nm})$ and gold $(200 \mathrm{~nm})$ sequentially. The electrodes are $1 \mathrm{~mm}$ wide with a $1 \mathrm{~mm}$ gap in between. To enable a controlled temperature loop, each sensor chip is glued using Aremco Ceramabond 571 on top of a ceramic heater substrate (Heraeus $\mathrm{GmbH}$, Germany) with an additional Pt-100 temperature sensor (Heraeus $\mathrm{GmbH}$, Germany). This device was mounted on top of a TO8-socket and connected to its pins using gold-wire bonding and silver glue (Epotek E3081). This setup limits the temperature that can be used within stable conditions to $300^{\circ} \mathrm{C}$. The final sensor is inserted into a flow cell which is attached to a gas mixing system. A brief description of this system can be found here [22]. A dry background mixture of $\mathrm{N}_{2}$ and $\mathrm{O}_{2}$ with a ratio of $80: 20 \mathrm{ml} / \mathrm{min}$ and a constant flow rate of $100 \mathrm{ml} / \mathrm{min}$ was used both as a carrier gas and purging gas. The nitrogen concentration was then adjusted when introducing a test gas to the gas flow. To test the influence of UV light, a UV LED (Sensor Electronic Technology Inc, S-T39B-F1-265-01-1-050) with a wavelength of $265 \mathrm{~nm}$ and an optical output power of $0.8 \mathrm{~mW}$ was used for some measurements. 


\section{Results and Discussion}

\subsection{Morphological and structural characterization}

\subsubsection{Atomic force microscopy}

To see that the surface decoration with $\mathrm{TiO}_{2}$ and $\mathrm{Fe}_{3} \mathrm{O}_{4}$ nanoparticles was successfully performed, AFM measurements were conducted before and after the deposition. Figure 1 (a) shows the distinct topography of an as-grown graphene layer with almost no roughness, but the characteristic steps from the $\mathrm{SiC}$ substrate after step-bunching upon the high temperature graphene growth. The topography after the additional deposition of iron oxide nanoparticles is shown in Figure 1 (b), showing monodispersed, spherical particles with diameters mostly in the range of $40-80 \mathrm{~nm}$, with a few larger aggregates.

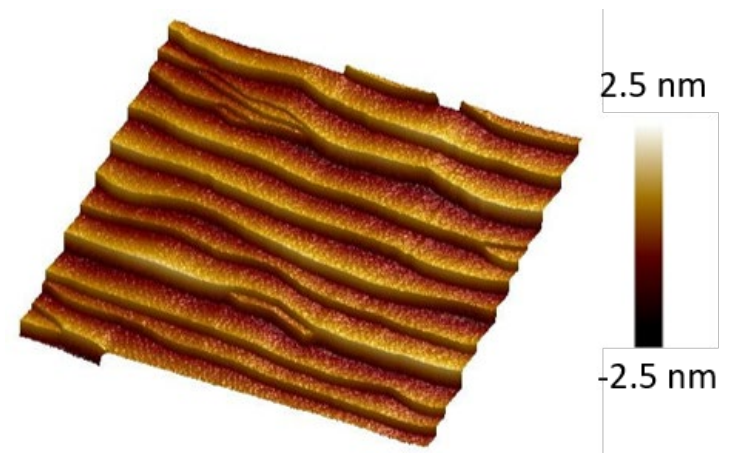

(a)

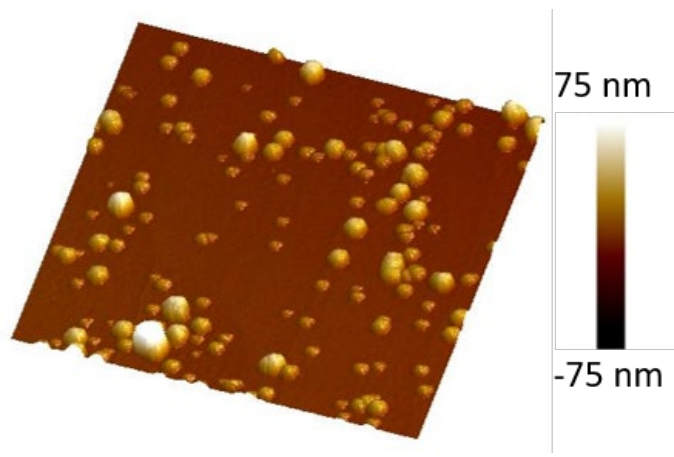

(b)

Figure 1:

AFM scans over an area of $5 \times 5 \mu m^{2}$ of (a) as-grown graphene and an area of $2 \times 2 \mu m^{2}$ (b) with $\mathrm{Fe}_{3} \mathrm{O}_{4}$ nanoparticles decorated on top of the graphene surface.

\subsubsection{Raman spectroscopy}

Raman spectroscopy measurements were also conducted before and after the deposition to ascertain the quality of the graphene and to study potential damage introduced through the nanoparticle deposition. The comparison between the as-grown graphene and graphene decorated with $\mathrm{Fe}_{3} \mathrm{O}_{4}$ nanoparticles is shown in Figure 2. The intensity scale is in arbitrary units and the signals were fitted to have the same maximum peak height for the G-peak at around $1610 \mathrm{~cm}^{-1}$. The black line corresponds to the Raman spectrum for an as-grown graphene reference which shows the typical $G$ and 2D peaks around $1610 \mathrm{~cm}^{-1}$ and $2737 \mathrm{~cm}^{-1}$, respectively [23]. The features starting in the range of $1280 \mathrm{~cm}^{-1}$ and extending into the G-peak are all related to the interfacial buffer layer between the graphene and the SiC substrate [23], and while they overlap with the position of the D peak they are not related to defects. It can be clearly seen that the spectra almost completely overlap, indicating that no damage was introduced into the graphene surface during the nanoparticle deposition. If damage is introduced to the graphene surface, the broad D peak around $1350 \mathrm{~cm}^{-1}$ would rise, indicating a rise of level of disorder and impurities. Furthermore, the 2D peak FWHM value of around $35 \mathrm{~cm}^{-1}$ is an additional indicator of the high level of monolayer graphene [24]. 


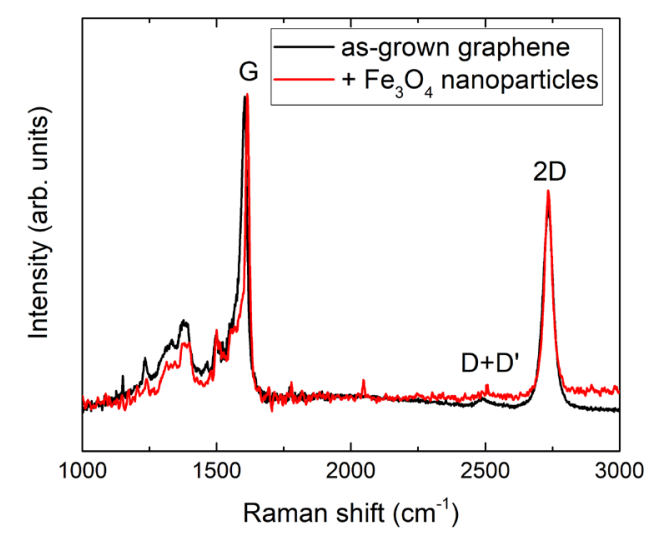

Figure 2:

Raman spectra of the graphene surface before and after the deposition of $\mathrm{Fe}_{3} \mathrm{O}_{4}$ nanoparticles on top.

\subsection{Gas measurements}

\subsubsection{Surface decoration}

After the verification that the graphene sensors were successfully decorated with nanoparticles and that the graphene structure is maintained, gas measurements were performed. It was already shown in earlier work that $\mathrm{TiO}_{2}$ nanoparticle decorated graphene sensors can be used for formaldehyde detection [16]. Here, the response of $\mathrm{Fe}_{3} \mathrm{O}_{4}$ nanoparticle decorated graphene is used for the detection of the even more toxic benzene. The sensor was exposed for 40 minutes towards 100, 50, 10 and $5 \mathrm{ppb}$ benzene at $150{ }^{\circ} \mathrm{C}$ in dry air, respectively. Each exposure was conducted twice and the final resistance signal was processed to show the data without drift. The sensor response is shown in Figure 3. The response is defined as Response $=\frac{R-R_{0}}{R_{0}}$, where $R$ is the saturated resistance signal and $R_{0}$ corresponds to the baseline resistance before the exposure. It can be clearly seen that the sensor response is distinct over the whole range and even an exposure towards only $5 \mathrm{ppb}$ benzene leads to a significant change of $7 \Omega$ or $0.3 \%$. Although this value is very small, it is no problem to detect it with the used setup, because the sensor has a very high signal to noise ratio of $35 \mathrm{~dB}$ towards $5 \mathrm{ppb}$ benzene, calculated based on a noise level of three times the standard deviation of the baseline signal. Since benzene has no safety level yet, due to difficulties in determining its toxicity at trace concentrations, the aim has to be to measure concentrations as low as possible. Indeed benzene has been shown to be carcinogenic at any concentration [25]. With a detection of $5 \mathrm{ppb}$ of benzene, we reach the range of safety level for other VOCs like formaldehyde. Moreover, it can be clearly seen that the detection limit is not yet reached and even concentrations in the sub-ppb level should be possible to detect with this sensor ( $5 \mathrm{ppb}$ is the lowest reliable concentration permitted by the experimental setup), making it even more promising for benzene monitoring. 


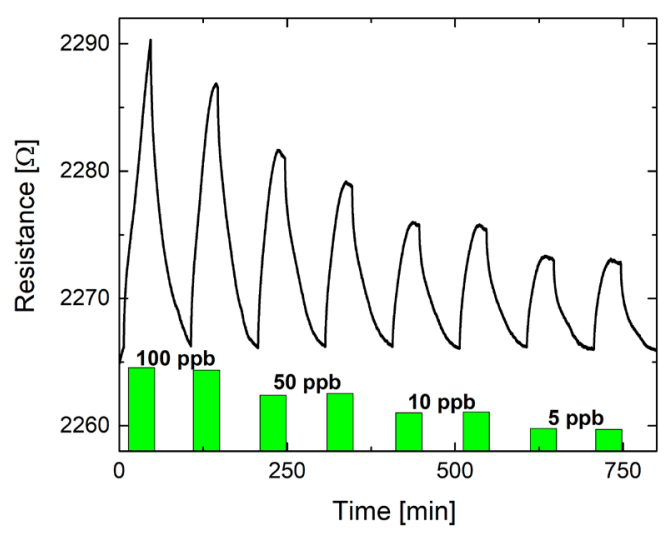

(a)

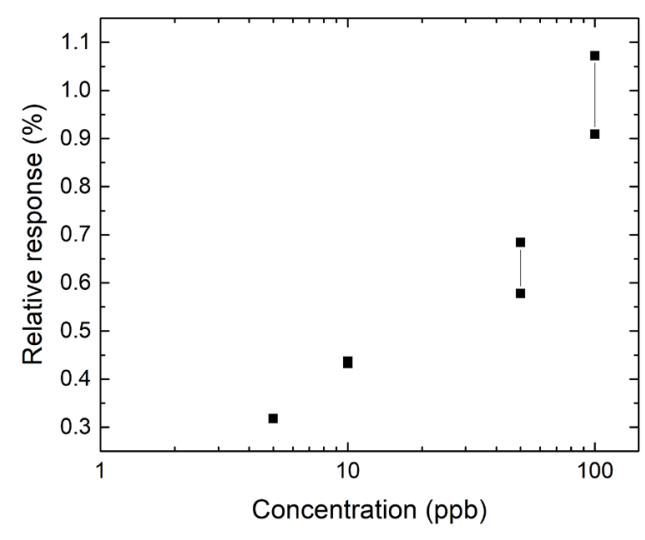

(b)

Figure 3: $\quad$ Sensor response (a) of $\mathrm{Fe}_{3} \mathrm{O}_{4}$ decorated graphene sensor at $150{ }^{\circ} \mathrm{C}$ towards different benzene concentrations of 100, 50, 10 and $5 \mathrm{ppb}$. (b) Shows the relative response versus benzene concentration for the exposures shown in (a).

\subsubsection{UV-light illumination}

UV light can be used to additionally change surface properties and enhance the sensing performance [18]. Especially for graphene, it was shown that UV light illumination can be attributed to cleaning of the surface, hence freeing active sites for target gas adsorption [26]. This is demonstrated on a $\mathrm{TiO}_{2}$ nanoparticle decorated graphene sensor detecting different levels of oxygen. Figure 4 shows the difference of the same sensor at $100{ }^{\circ} \mathrm{C}$ and exposed to a varying oxygen percentage between 15 and $2 \%$ (from a baseline of $20 \%$ ), without (a) and with (b) UV light illumination throughout the whole measurement. Not only does the UV light introduce a change in the baseline resistance of about $-270 \Omega$, but it can also be clearly seen that there is almost no distinct change of resistance (response) without UV light, whereas the measurement with UV illumination shows the typical shape of a gas sensor response. Moreover, the desorption process is enhanced under the illumination, decreasing the time needed for the sensor to get back to its baseline after the gas exposure, making it faster and more effective. Indeed the low response without UV is likely due to an oxygen saturated surface in the background of $20 \% \mathrm{O}_{2}$; UV illumination promotes oxygen desorption, leading to an increased dynamic range for oxygen detection.

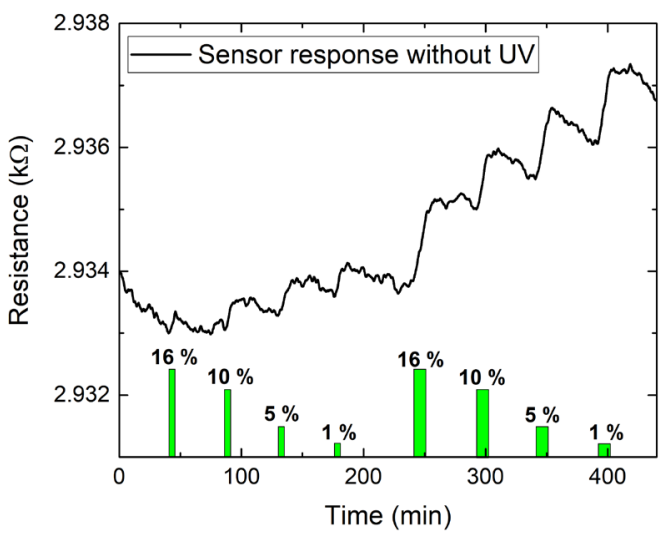

(a)

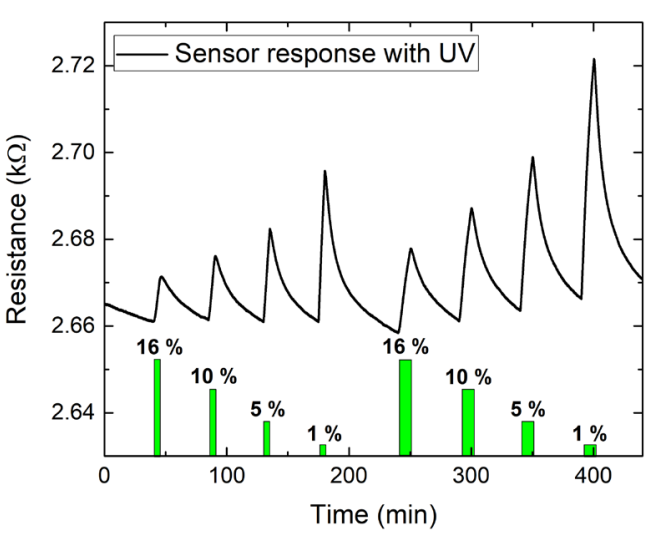

(b)

Figure 4: $\quad$ Sensor response of $\quad \mathrm{TiO}_{2}$ nanoparticle decorated graphene sensor at $100{ }^{\circ} \mathrm{C}$ towards a change of oxygen from a background of $20 \%$ to between 16 and $1 \%$ (a) without and (b) with UV light illumination. 


\subsubsection{Signal processing}

As a final step, smart data processing is evaluated towards decreasing the time constants needed for a good sensor response. It was already shown above that the introduction of UV light enhances the sensor, but still, time constants ( $(90)$ of around $1600 \mathrm{~s}$ for adsorption are needed before the sensor signal saturates (see Figure 5 (a) blue line). In this measurement, oxygen was variated between 16 and $1 \%$ (from a baseline of $20 \%$ ) with a varying exposure time of 5 and 10 minutes while the sensor was used at $200{ }^{\circ} \mathrm{C}$ and UV light was applied. Instead of waiting until the sensor response reaches a saturated level, we introduce the use of the time derivative signal. This signal is equal to the slope of the change in resistance during the measurement and as one can clearly see on the red line with the right $y$-axis in Figure $5(a)$, also the derivative signal exhibits distinct peaks for the gas exposures with not much change after this peak hits its maximum. After smoothening the data with a moving average filter, the time constant for the derivative signal peak can be calculated. The improvement in response time resulting from having to expose the sensor just the time needed to reach the maximum of the derivative signal is in this case up to a factor of 25. For example the sensor needs $1540 \mathrm{~s}$ to reach $\mathrm{\tau} 90$ (90\% of the saturated response), whereas it only needs 60 s to reach $t 90$ of the derivative signal, for a change to $10 \%$ oxygen. Moreover, the peak height of the derivative signal also corresponds to the concentration and could consequently be used as a sensor response. However, the desorption peak heights do not correspond that clearly to the concentration. Because of the smaller time constants, the actual sensor response does not have to be saturated when using the slope instead of the absolute value of resistance.

Figure 5 (b) shows what happens when the actual sensor signal is not fully recovered before the next concentration is introduced. Not only the derivative response is very stable for the different concentrations, the response magnitude is also stable if the raw sensor signal is not fully recovered, but the exposure time exceeds the time needed for the derivative signal to reach its maximum.

This behavior was also found for other sensing layers and other test gases, leading to a huge improvement when it comes to time constants. This can be especially useful for applications where short sampling times are needed, for example air quality monitoring.

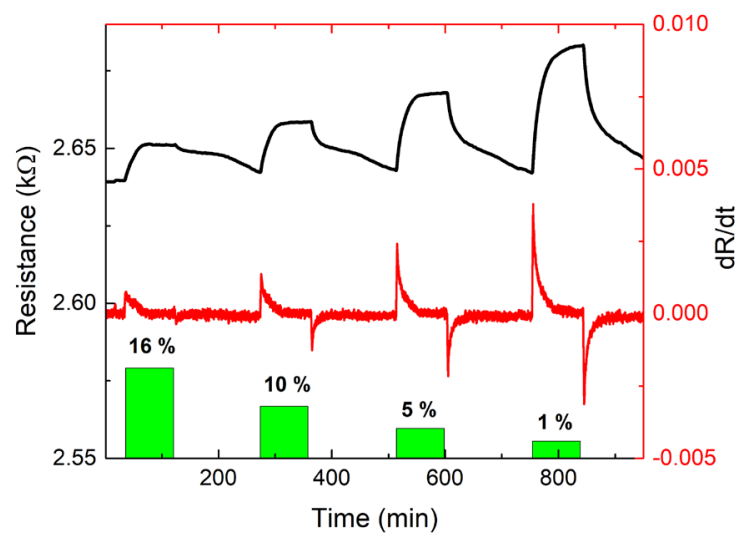

(a)

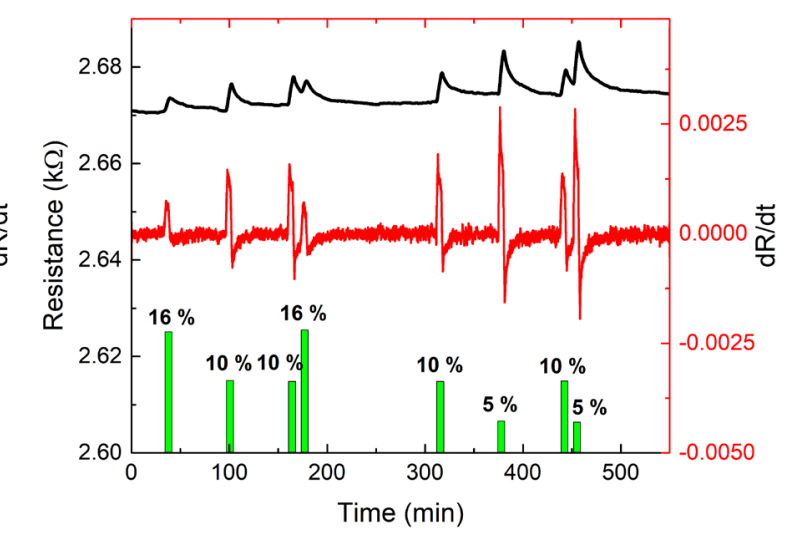

(b)

Figure 5: $\quad$ (a) Sensor responses (blue line with left $y$-axis) and time derivative of the responses (red line with right $y$ axis) of $\mathrm{TiO}_{2}$ nanoparticle decorated graphene sensor at $200{ }^{\circ} \mathrm{C}$ towards a change of oxygen between 16 and $1 \%$ (from a baseline of $20 \%$ ) with UV light illumination. (b) Response of the raw sensor signal and the time derivative signal upon incomplete sensor signal recovery between changes in gas concentration. 


\subsubsection{Sensor design implications}

The sensor design described in this study is completely scalable: The use of epitaxial graphene on SiC allows wafer scale production of devices that can be processed directly on the as-grown material as the graphene is grown on a semi-insulating substrate. The sputter-based technique used for nanoparticle deposition can be done on several wafers in one run, and the UV-LED is an off the shelf product which can be easily integrated into a sensor layout.

\section{Conclusion}

The successful decoration of epitaxial graphene sensors with metal oxide nanoparticles $\left(\mathrm{TiO}_{2}\right.$ and $\mathrm{Fe}_{3} \mathrm{O}_{4}$ ) using hollow cathode sputtering without damaging the graphene surface was shown using AFM and Raman. With the iron oxide nanoparticle decorated graphene sensor, it was easily possible to detect benzene down to $5 \mathrm{ppb}$ making it a promising sensor setup for ultra-high sensitivity gas sensors for VOC detection. It was also shown that with illumination with UV light of the titanium oxide nanoparticle decorated graphene sensor during the gas exposure, sensitivity towards a change of oxygen could be created where almost none could be seen without the UV light. As a final improvement, the time derivative signal was introduced to show that time constants can be decreased by at least one order of magnitude and that it might be used as a sensor response because it exhibits distinct changes corresponding to the applied gas concentration even if the actual sensor response is not saturated or if a new exposure takes place before the sensor had time to fully relax. All these three means of sensor improvement show very promising results for future sensor setups and will be investigated further to optimize sensitivity, selectivity and time constants of epitaxial graphene based gas sensors.

\section{Acknowledgements}

The authors would like to thank Anita Lloyd Spetz for discussion and support. Moreover, we thank the Swedish Foundation for Strategic research (SSF) for financial support through the grant GMT140077. We acknowledge support from the Swedish Government Strategic Research Area in Materials Science on Functional Materials at Linköping University (Faculty Grant SFO-Mat-LiU \# 2009-00971). This work has been supported by The Centre in Nano science and technology (CeNano) through the project "Graphene-nanoparticle hybrid gas sensor"

\section{Data availability}

The raw/processed data required to reproduce these findings cannot be shared at this time due to technical or time limitations. The authors will happily share data with anyone interested on request.

\section{References}

[1] World Health Organisation, Indoor Air Quality: Organic Pollutant, Copenhagen (1989)

[2] Council of the European Union, Official Journal of the European Communities, L152, 1-43 (2008)

[3] World Health Organisation, Inheriting a sustainable world? Atlas on children's health and the environment, Geneva, ISBN 9789241511773 (2017)

[4] BCC Research, Portable Gas Detection Systems: Global Markets (2016) 
[5] A. C. Ferarri et al., Science and technology roadmap for graphene, related two-dimensional crystals, and hybrid systems, Nanoscale 7, 4598 (2015)

[6] S. S. Varghesea, S. Lonkara, K.K. Singhc, S. Swaminathan, and A. Abdalad, Recent advances in graphene based gas sensors, Sensors and Actuators B 218, 160-183 (2015)

[7] R. Pearce, J. Eriksson, T. lakimov, L. Hultman, A. Lloyd Spetz, and R. Yakimova, On the differing sensitivity to chemical gating of single and double layer epitaxial graphene explored using Scanning Kelvin Probe Microscopy, ACS Nano 7 (5), 4647-4656 (2013)

[8] C. Virojanadara, M. Syväjarvi, R. Yakimova, and L. I. Johansson, Phys. Rev. B 78, 245403 (2008)

[9] A. Tzalenchuk, S. Lara-Avila, A. Kalaboukhov, S. Paolillo, M. Syväjärvi, R. Yakimova, O. Kazakova, T. J. B. M. Janssen, V. Fal'ko and S. Kubatin, Towards a quantum resistance standard based on epitaxial graphene, Nat. Nano 5, 186-189 (2010)

[10] Jens Eriksson, Donatella Puglisi, Yu Hsuan Kang, Rositza Yakimova, and Anita Lloyd Spetz, Adjusting the electronic properties and gas reactivity of epitaxial graphene by thin surface metallizations, Physica B 439, 105-108 (2014)

[11] J. Eriksson, D. Puglisi, C. Strandqvist, R. Gunnarsson, S. Ekeroth, I. G. Ivanov, U. Helmersson, K. Uvdal, R. Yakimova, and A. Lloyd Spetz, Modified Epitaxial Graphene on SiC for Extremely Sensitive and Selective Gas Sensors, Materials Science Forum 858, 1145-1148 (2016)

[12] F.-L. Meng, H.-H. Li, L.-T. Kong, J.-Y. Liu, Z. Jin, W. Li, Y. Jia, J.-H. Liu, and X.-J. Huang, Parts per billion-level detection of benzene using $\mathrm{SnO} 2 /$ graphene nanocomposite composed of sub- $6 \mathrm{~nm} \mathrm{SnO2}$ nanoparticles. Analytica Chimica Acta, 736, 100-107 (2012)

[13] S. Mao, S. M. Cui, G. H. Lu, K. H. Yu, Z. H. Wen, and J. H. Chen, Tuning gas-sensing properties of reduced graphene oxide using tin oxide nanocrystals, J. Mater. Chem. 22, 11009-11013 (2012)

[14] S. Liu, B. Yu, H. Zhang, T. Fei, and T. Zhang, Enhancing NO2 gas sensing performances at room temperature based on reduced graphene oxide-ZnO nanoparticles hybrids, Sens. Actuators B: Chem. 202, 272-278 (2014)

[15] G. Singh, A. Choudhary, D. Haranath, A. G. Joshi, N. Singh, S. Singh, and R. Pasricha, ZnO decorated luminescent graphene as a potential gas sensor at room temperature, Carbon 50, 385-394 (2012)

[16] A. Esfandiar, S. Ghasemi, A. Irajizad, O. Akhavan, and M.R. Gholami, The decoration of $\mathrm{TiO} 2 /$ reduced graphene oxide by Pd and Pt nanoparticles for hydrogen gassensing, Int. J. Hydrogen Energy 37, 15423-15432 (2012)

[17] R. Gunnarsson, U. Helmersson, and I. Pilch, Synthesis of titanium-oxide nanoparticles with size and stoichiometry control. Journal of Nanoparticle Research, 17 (9), 1-11 (2015)

[18] M. Kodu, A. Berholts, T. Kahro, T. Avarmaa, A. Kasikov, A. Niilisk, H. Alles, and R. Jaaniso, Highly sensitive NO2 sensors by pulsed laser deposition on graphene, Applied Physics Letters 109, 113108 (2016)

[19] A. Schütze, T. Baur, M. Leidinger, W. Reimringer, R. Jung, T. Conrad, T. Sauerwald, Highly Sensitive and Selective VOC Sensor Systems Based on Semiconductor Gas Sensors: How to? Environments, 4, 20 (2017)

[20] R. Yakimova, C. Virojanadara, D. Gogova, M. Syväjärvi, D. Siche, K. Larsson, and L. I. Johansson, Analysis of the formation conditions for large area epitaxial graphene on SiC substrates, Mater. Sci. For. 565-568, 645 (2010) 
[21] I. Pilch, D. Söderström, M. I. Hasan, U. Helmersson, and N. Brenning, Fast growth of nanoparticles in a hollow cathode plasma through orbit motion limited ion collection. Applied Physics Letters, 103 (19), 193108 (2013)

[22] A. Lloyd Spetz and M. Andersson, Technology and Application Opportunities for SiC-FET Gas Sensors, Solid State Gas Sensors - Industrial Application, 281 (2012)

[23] D. S. Lee, C. Riedl, B. Krauss, K. von Klitzing, U. Starke, and J. H. Smet, Raman Spectra of Epitaxial Graphene on $\mathrm{SiC}$ and of Epitaxial Graphene Transferred to $\mathrm{SiO}_{2}$, Nano Letters 8 (12), 4320-4325 (2008)

[24] A. C. Ferrari, Raman spectroscopy of graphene and graphite: Disorder, electron-phonon coupling, doping and nonadiabatic effects, Solid State Communications 143, 47-57 (2007)

[25] WHO Guidelines for Indoor Air Quality: Selected Pollutants, ISBN 9789289002134 (2010)

[26] A. Berholts, T. Kahro, A. Floren, H. Alles and R. Jaaniso, Photo-activated oxygen sensitivity of graphene at room temperature, Applied Physics Letters, 105 (16), 163111 (2014) 